\title{
HISTORIA DE LA ARTROPLASTIA TOTAL DE CADERA
}

\author{
E. Álvarez*, E. Villanueva \\ Hospital Universitario Dr. José E. González, Universidad Autónoma de Nuevo León \\ * contacto@medicinaarticular.com, dedaloz@gmail.com \\ * E-Mail: jcorona@ ciateq.mx
}

\begin{tabular}{ll}
\hline PALABRAS CLAVE & RESUMEN \\
\hline $\begin{array}{l}\text { Artroplastia } \\
\text { Badera }\end{array}$ & $\begin{array}{l}\text { La patología articular que incluye la osteoartrosis en conjunto con la } \\
\text { osteonecrosis, son patologías cuyo común denominador es la destrucción } \\
\text { John Charnley }\end{array}$ \\
articular nativa y que es un problema de salud altamente prevalente por la \\
naturaleza de la evolución humana. Desde la primera mitad del siglo XIX, se \\
entendió la necesidad de tratamiento para la patología debido a la alta \\
morbilidad que esta conlleva. Todos los tratamientos utilizados desde ese \\
tiempo han buscado devolver la funcionalidad y minimizar el dolor. Al inicio se \\
utilizaron tratamientos rudimentarios como la extirpación articular de Henry \\
Park, sin embargo, la evolución científica nos ha llevado al desarrollo de \\
técnicas menos invasivas, que buscan mejorar la condición física del paciente. \\
La artroplastia total de cadera es un avance de grandes dimensiones dentro de la \\
ortopedia moderna representando la idea básica de reemplazar una articulación \\
destruida. Este procedimiento tan utilizado en la actualidad ha sufrido grandes \\
cambios desde sus inicios en 1962 cuando John Charnley la describió. Incluso \\
en la actualidad existen diferentes materiales que dan resultados diferentes en \\
una artroplastia y por ello se desarrollan indicaciones para cada uno de estos. \\
Los pares de fricción han evolucionado en búsqueda de aquel que sea resistente, \\
biomecánicamente funcional y con la menor tasa de complicaciones posible.
\end{tabular}

\section{HISTORY OF TOTAL HIP ARTHROPLASTY}

\begin{tabular}{ll}
\hline KEYWORDS & ABSTRACT \\
\hline Arthroplasty & Joint pathology or osteoarthrosis together to osteonecrosis are highly prevalent \\
Hip & diseases by the nature of human evolution. Since the first half of the 19th century, \\
Biomaterials & the need for treatment for the pathology was understood due to the high morbidity \\
John Charnley & that it entails. All the treatments designed since that time have been in search of \\
Ceramics & returning the functionality to the patient and minimizing their pain. Initially, \\
& rudimentary treatments were used, such as Henry Park's joint removal, however, \\
& scientific evolution has led us to develop less invasive techniques that seek to \\
& improve the patient's physical condition. Total hip arthroplasty is a major advance \\
& in modern orthopedics representing the basic idea of replacing a destroyed joint. \\
& This widely used procedure today has undergone major changes since its inception \\
& in 1962 when John Charnley described it. Even today, different materials change \\
the results of arthroplasty and because of this they developed indications for each \\
of these. Friction pairs have evolved in search of those that are resistant, \\
biomechanically functional, and with the lowest possible complication rate.
\end{tabular}


E. Álvarez, E. Villanueva

Revista Colombiana de Materiales, ISSN 2256-1013, No 17, pp. 3-13, 2021. https://doi.org/10.17533/udea.rcm.n17a01

\section{OSTEONECROSIS DE LA CABEZA FEMORAL}

La osteonecrosis de la cabeza femoral es una patología con etiología desconocida en la cual la muerte de las células óseas y la destrucción articular es el resultado final de un conjunto de mecanismos como isquemia, toxicidad celular directa y diferenciación alterada de las células mesenquimales [1]. Se presentan entre 20000 y 30000 casos nuevos cada año en Estados Unidos y aunque se han descrito múltiples tratamientos en los cuales se incluyen la descompresión central, el uso de injertos vascularizados o incluso osteotomías óseas, la artroplastia total de cadera, es considerada por muchos autores como el tratamiento más razonable [2-11]. La osteonecrosis de la cabeza femoral representa el 10-12\% de los diagnósticos en pacientes en los cuales se realiza una artroplastia total de cadera $[12,13]$.

\section{ARTROPLASTIA CADERA}

La artroplastia total de cadera es un avance de grandes dimensiones dentro de la ortopedia moderna representando la idea básica de reemplazar una articulación destruida o disfuncional para minimizar el dolor y preservar la funcionalidad [14]. Desde sus inicios, la reconstrucción articular de cadera ha presentado una constante evolución desde el siglo XIX hasta la actualidad, comenzando con métodos rudimentarios que se han ido modificando de manera gradual.

La patología articular u osteoartrosis es una enfermedad altamente prevalente por la naturaleza de la evolución humana, incluso siendo evidenciada en cadáveres de Homo sapiens y Homo sapiens neanderthalensis en excavaciones [15].

El estudio de una patología tan frecuente y con la morbilidad que la osteoartrosis conlleva, se inició hacia la primera mitad del siglo XIX. La extirpación de las articulaciones era un método que se practicaba deliberadamente. Henry Park, quien trabajaba en The Royal Infirmary, describió la técnica como: [16]

“...la total extirpación de la articulación o de los huesos que forman la articulación, con la mayor parte o totalidad del ligamento capsular, obteniendo la curación a través de la formación de callo..."

Anthony White en el Westminster Hospital de Londres realizó la primera artroplastia por excisión en 1821, sin embargo, no realizó ningún reporte. La comunidad médica lo reconoció en el Lancet:

“...excisó la cabeza, cuello y trocánteres del fémur, con el paciente sobreviviendo doce años, muriendo por consunción."

John Rhea Barton en Filadelfia realizó la primera osteotomía en una cadera anquilosada en 1826. Fue un procedimiento sin anestesia y realizado en siete minutos. A los veinte días manipuló la extremidad para provocar una reacción fibrosa en los extremos del hueso, formando así una pseudoartrosis [17]. El paciente pudo deambular razonablemente con un bastón tres meses posteriores al procedimiento, sin embargo, a seis años, toda la movilidad se encontraba perdida. Barton demostró de esta manera que la movilidad previene la fusión del hueso [18]. 
E. Álvarez, E. Villanueva

Revista Colombiana de Materiales, ISSN 2256-1013, No 17, pp. 3-13, 2021. https://doi.org/10.17533/udea.rcm.n17a01

Posteriormente, aparecieron las artroplastias de interposición con Léopold Ollier en Lyon, Francia, hacia 1885. Ollier describió la colocación de tejido adiposo en articulaciones sin infección, sin embargo, su eficacia fue muy limitada debido a que el material interpuesto no era fijado. Chlumsky en Alemania probó con una gama más amplia de tejidos para interposición: músculo, celuloide, placas de plata, goma, zinc, pyrex, hueso descalcificado, cera y magnesio, este último provocando la consolidación y fusión de la articulación.

Themistocles Glück en 1891 fue el pionero en cuanto a la fijación de los implantes. Utilizó una esfera de marfil y una copa que fijó con tornillos de níquel al hueso [19]. Eventualmente, experimentó con una mezcla de yeso, pumita pulverizada con resina. Sin embargo, este esfuerzo falló debido a la resorción ósea [20].

Entrando al siglo XX, Murphy y Lexer en Münich describieron la interposición de fascia lata, y William Steven Baer popularizó la artroplastia de vejiga porcina en 1928 en el Johns Hopkins de Baltimore [21,22].

Sir Robert Jones utilizó láminas de oro para cubrir la cabeza femoral reconstruida. Veinte años después reportó que el paciente mantenía movilidad efectiva en la articulación. Este fue el seguimiento más largo reportado hasta ese punto, en la historia de la artroplastia.

Fue hasta que Marius Smith-Petersen de Boston, Massachusetts en 1923 utilizó una prótesis de molde en la cual utilizó material sintético como interposición. Elaboró una prótesis de vidrio que colocó entre la cabeza femoral y el acetábulo.
Además, describió el abordaje anterior para este tipo de procedimientos. Posteriormente experimentó con celuloide, Bakelite y pyrex. En 1937, su dentista sugirió utilizar Vitallium, lo cual lo llevó a colocar 500 prótesis de este material con buenos resultados clínicos. Este dispositivo proveyó los primeros resultados predecibles en una artroplastia de cadera [23]. En 1923, Gathorne Robert Girdlestone popularizó la resección de la cabeza femoral, especialmente por tuberculosis e infección, en Reino Unido [24]. Este procedimiento, aunque arcaico, ocasionalmente es utilizado en pacientes pediátricos y tiene un uso limitado en adultos, generalmente como último recurso en la artroplastia fallida $[16,18,24,25]$.

Austin Moore colocó la primera prótesis de metal en el Johns Hopkins en 1940, la cual tenía un ángulo de $135^{\circ}$ y vástago largo [26]. Posteriormente, en 1952 perfeccionó con Böhlman el modelo, con un diseño fenestrado. Frederick Röeck Thompson desarrolló una prótesis de Vitallium con un cuello acampanado y un vástago recto en 1950 [27]. Estos diseños posteriormente se volvieron ampliamente utilizados, especialmente en pacientes con fractura del cuello femoral, no obstante tienden a fallar tempranamente debido a la presión excesiva en acetábulo o componente acetabular. En 1938, Wiles en el Middlesex Hospital de Londres desarrolló la primera artroplastia no cementada fijada con tornillos [28].

Haboush en Nueva York y McKee en Inglaterra en 1940 desarrollaron de igual manera prótesis y experimentaron con cemento acrílico utilizado en la industria dental. A partir de 1950, McKee desarrolló 
E. Álvarez, E. Villanueva

Revista Colombiana de Materiales, ISSN 2256-1013, No 17, pp. 3-13, 2021. https://doi.org/10.17533/udea.rcm.n17a01

una prótesis de Thompson articulada con una copa "en garra" atornillada al acetábulo. Peter Ring de igual manera experimentó con prótesis de articulación metal-metal en 1964. Este tuvo buenos resultados con hasta el $97 \%$ de implantes presentando una sobrevida de hasta 17 años [18]. Muller en 1957 desarrolló una copa plástica acetabular en combinación con un implante y cabeza de $32 \mathrm{~mm}$ de cromo-cobalto-molibdeno. Sivash, durante ese mismo tiempo utilizó implantes metálicos tanto en acetábulo como en fémur con un inserto de polietileno entre ellos [25,29].

John Charnley del Wrightington Hospital, en 1962, realizó un reemplazo articular histórico utilizando implantes de baja fricción con un polietileno de alta densidad entre los componentes metálicos y estabilizándolos con cemento acrílico. Esta combinación de materiales mostró buenos resultados. El diseño de Charnley constaba de un vástago femoral con cabeza de acero inoxidable, y un componente acetabular de polietileno, fijados al hueso mediante cemento acrílico [25,29].

Inicialmente se utilizaba una cabeza femoral de 22,2 $\mathrm{mm}$ para una fricción reducida contra el componente acetabular, sin embargo, se luxaba fácilmente. Müller desarrolló cabezas más grandes, mejorando la estabilidad, pero el desgaste que presentaban hizo que la tasa de fallas aumentara. Otra de las contribuciones de Sir John Charnley fue el uso de cemento óseo de polimetilmetacrilato (PMMA) para fijar los componentes al hueso. La artroplastia de baja fricción de Charnley persiste, aunque con pequeñas modificaciones, en el mundo de la artroplastia de cadera $[25,29,30]$.
En 1970, Boutin introdujo la articulación de cerámica-cerámica, y cuatro años después Heinz Mittlemeier colocó un vástago metálico con copa y cabeza de cerámica $\left(\mathrm{Al}_{2} \mathrm{O}_{3}\right)$. Este implante es considerado por muchos cirujanos ortopédicos como el más efectivo y resistente $[25,29,30]$.

Ling y Lee diseñaron en 1970 el vástago Exeter, de perfil cónico, pulido, el cual funcionaría como una cuña al introducirse al fémur, confiriendo una mayor estabilidad durante un mayor periodo de tiempo $[14,25,29,30]$. Este fue colocado por primera vez en el Princess Elizabeth Orthopaedic Hospital en Exeter, Inglaterra. Este implante no se unía en la interfaz del metal y cemento, por tanto, tiene cierto micromovimiento, impactándose cada vez más dentro del fémur, conforme avanza el tiempo $[25,29,30]$.

Durante la década de los 80, Galante introdujo los implantes no cementados press-fit, que tienen la característica de introducirse a presión tanto en el acetábulo como en el canal femoral $[25,29]$.

A partir de la última década del siglo XX, se han propuesto nuevas innovaciones al reemplazo total de cadera, como lo es la cirugía mínimamente invasiva, con la ventaja de menor sangrado y rehabilitación temprana. Derek McMinn desarrolló en 1997, la cadera Birminham, utilizada en la técnica llamada resurfacing, la cual consiste en sustituir el cartílago de la cabeza femoral y acetábulo con implantes metálicos. Con el advenimiento tecnológico la cirugía asistida por computadora y robótica ha ido tomando cada vez más fuerza entre los cirujanos ortopédicos, sin embargo, esta técnica 
E. Álvarez, E. Villanueva

Revista Colombiana de Materiales, ISSN 2256-1013, No 17, pp. 3-13, 2021. https://doi.org/10.17533/udea.rcm.n17a01

sigue en desarrollo para su perfeccionamiento.

\subsection{Tribología}

La durabilidad de la prótesis de cadera es un área de amplio interés entre los cirujanos ortopédicos debido al incremento en la esperanza de vida de los pacientes, la colocación de prótesis en pacientes cada vez más jóvenes y por consiguiente un nivel de actividad más alto.

Las tasas de desgaste de 75-250 $\mu \mathrm{m} / \mathrm{año}$ en la superficie del polietileno llevan a una osteolisis que compromete la supervivencia de la prótesis, su estabilidad $\mathrm{y}$, finalmente, su durabilidad [31,32].

Los pares de fricción se dividen en 2 tipos:

\section{- Duro-suave (D-S)}

El componente suave siempre hace referencia al acetábulo e incluye el Polietileno de Ultra Alto Peso Molecular (PEUAPM) y el PUAPM con enlaces cruzados.

\section{- Duro-duro (D-D)}

Incluye aleaciones de metal (cromocobalto) y cerámicas

\subsubsection{Pares de fricción $D-S$}

\subsubsection{Metal-Polietileno de enlaces cruzados}

Durante las últimas cinco décadas el par de fricción más aceptado es el de cabeza metálica de cromo-cobalto con componente acetabular de PEUAPM, llegando a ser considerado el estándar. Sin embargo, el mayor problema del
PEUAPM, cuando se utiliza con cabezas metálicas es el desgaste prematuro, el cual está relacionado al material, esterilización, geometría del implante y su limitado tiempo de almacenamiento.

Inicialmente se pensó que la osteolisis del hueso periprotésico era dada por la reacción ósea al cemento (PMMA), lo que llevó a llamarle la "enfermedad del cemento" [33,34]. Conforme avanzó el tiempo, se identificó que las partículas del desgaste del PEUAPM producen una respuesta a cuerpo extraño, desencadenando una cascada inflamatoria mediada por macrófagos, con la consiguiente liberación de citocinas proinflamatorias, y mediadores de la resorción ósea. El efecto final era el aflojamiento aséptico del implante [3537]. Se demostró que ésta era realmente la causa de falla de la prótesis y no la famosa "enfermedad del cemento".

De acuerdo con observaciones, la osteolisis se presenta más frecuentemente con tasas de desgaste mayores de $0,1 \mathrm{~mm} / \mathrm{año}$ e infrecuente cuando estas son menores de 0,05 mm/año [38,39]. En 1998, para mejorar la tasa de desgaste, se comenzó a utilizar el PEUAPM de enlaces cruzados utilizando radiación gamma y tratamiento térmico, incrementando así, la resistencia a la oxidación de PEUAPM [40-44]. Sin embargo, esto trajo nuevos problemas, como la formación de radicales libres del oxígeno y la oxidación del polietileno posterior al tratamiento térmico. En la actualidad, estos problemas se abordan adicionando vitamina $\mathrm{E}$ o el recocido secuencial para saturar los radicales libres [45-47]. 
E. Álvarez, E. Villanueva

Revista Colombiana de Materiales, ISSN 2256-1013, No 17, pp. 3-13, 2021. https://doi.org/10.17533/udea.rcm.n17a01

\subsubsection{Cerámica-Polietileno de enlaces cruzados}

El uso de cabeza de cerámica con polietileno de enlaces cruzados ha ido ganando aceptación poco a poco ya que se ha demostrado una tasa de desgaste menor en un 10-50 \% [48]. Existen varias ventajas de la cerámica:

\section{Menor coeficiente de fricción} comparado con metal-metal.

2. Mejor lubricación en la articulación.

3. El residuo de desgaste liberado por la cerámica es químicamente inerte.

4. Menor susceptibilidad a la formación de rayaduras $[49,50]$.

\subsubsection{Pares de fricción $D-D$}

La formación de partículas en la utilización de polietileno llevó a utilizar prótesis con los pares de metal-metal y cerámica-cerámica. Este tipo de técnicas generan mucho menos partículas de desgaste y estas son de un menor tamaño $(0,015-0,12 \mu \mathrm{m})$, mientras que para que osteolisis se produzca, se necesitan partículas mucho mayores $(0,2-7 \mu \mathrm{m})$ [51].

\subsubsection{Resurfacing Metal-metal}

La técnica de resurfacing parece ser la mejor técnica de artroplastia debido a la mínima cantidad de hueso perdido con una estabilidad excelente. D.McMinn y R. Treacy en los años noventa, desarrollaron el diseño Birmingham para resurfacing, el cual mejoró el rango de movimiento, la estabilidad, resistencia, además de un importante mantenimiento del hueso nativo y por tanto una facilidad para sustituir el dispositivo en caso de fallo; sin embargo, existe inquietud respecto a la elevada cantidad de iones de cobalto y cromo en sangre, lo que se ha asociado a riesgo de cáncer, pseudotumores y lesiones vasculíticas [52].

A pesar de que se sigue utilizando bajo un estricto control y seguimiento, las técnicas de resurfacing asocian un alto índice de falla, sobre todo en pacientes del género femenino, con obesidad y componentes femorales de menor tamaño.

\subsubsection{Prótesis Metal-metal}

Con el éxito a corto plazo del resurfacing se decidió utilizar el par de metal-metal en componentes femorales utilizando un vástago con mayor tamaño de cabeza femoral, aumentando el radio cabezacuello. Esto mejora la excursión de los componentes y confiere mayor estabilidad al dispositivo, haciéndola una excelente opción para revisar prótesis luxadas.

Además de las complicaciones relacionadas al resurfacing, con este tipo de prótesis se empezó a observar corrosión en la unión del vástago a la cabeza de la prótesis, acuñando el término de truniosis. Estas complicaciones, las cuales asociaban una alta tasa de fallo, hicieron que este par de fricción haya sido abandonado en los últimos años.

\subsubsection{Cerámica-cerámica}

Después de la introducción de la cerámica en 1960 por Boutin, esta ha ido mejorando sobretodo en la susceptibilidad a la fractura [53]. Su mayor resistencia y dureza (formación de partículas nanométricas), la propiedad de ser inertes biológicamente, menores tasas de desgaste y una mejor lubricación hacen de la cerámica una excelente opción a largo plazo. Esto le provee a la cerámica una 
supervivencia a largo plazo excelente, siendo una muy buena opción en pacientes jóvenes y activos.

Existen dos tipos de cerámica, zirconia y alúmina. La utilización de la alúmina se ha preferido siempre sobre la zirconia, sin embargo, la nueva generación de cerámicas incorpora una mezcla de zirconia estabilizada con itria (25\%), una matriz de alúmina $(75 \%)$ y se refuerza con óxido de cromo $(<1 \%)$ [54].

Aunque se prefieran en los pacientes jóvenes debido a su durabilidad, y a sus propiedades, no están exentos de complicaciones como ruidos a la movilidad, fracturas de la superficie de apoyo y fragmentación durante la colocación. Estas complicaciones se asocian a la posición incorrecta del componente acetabular, cabezas femorales pequeñas y pobre técnica quirúrgica $[55,56]$.

\subsection{Futuro de los pares de fricción}

- Cabezas femorales de cerámica con componente acetabular metálico: menor desgaste y mucha menor generación de partículas de metal.

- Oxinium: Zirconia con oxidium, la cual reduce el riesgo de fractura de la cerámica.

- Adición de nitruro de silicio $\left(\mathrm{Si}_{3} \mathrm{~N}_{4}\right)$ : confiere un menor coeficiente de fricción $(0,001$ en comparación con 0,08 de la cerámica tradicional), mayor resistencia a fractura, flexibilidad, y resistencia a la degradación térmica.

- $\quad$ Prevención del stress-shielding: la utilización de copas reforzadas con fibra de carbón para prevenir el desgaste óseo retroacetabular.

\section{AGRADECIMIENTOS}

Agradecimientos al Consejo Nacional de Ciencia y Tecnología de México CONACYT a través del proyecto 313990 "Simposio Nacional de Prótesis Ortopédicas: El estatus Actual".

\section{REFERENCIAS}

[1] Zalavras CG, Lieberman JR. Osteonecrosis of the femoral head: evaluation and treatment. JAAOS-Journal Am Acad Orthop Surg. 2014;22(7):455464.

[2] Marker DR, Seyler TM, Ulrich SD, Srivastava S, Mont MA. Do modern techniques improve core decompression outcomes for hip osteonecrosis? Clin Orthop Relat Res. 2008;466(5):10931103.

[3] Markel DC, Miskovsky C, Sculco TP, Pellicci PM, Salvati EA. Core decompression for osteonecrosis of the femoral head. Clin Orthop Relat Res. 1996;323:226-233.

[4] Mont MA, Einhorn TA, Sponseller $\mathrm{PD}$, Hungerford DS. The trapdoor procedure using autogenous cortical and cancellous bone grafts for osteonecrosis of the femoral head. J Bone Joint Surg Br. 1998;80(1):56-62.

[5] Mont MA, Fairbank AC, Krackow KA, Hungerford DS. Corrective osteotomy for osteonecrosis of the femoral head. The results of a long-term follow-up study. JBJS. 1996;78(7):1032-1038.

[6] Phemister DB. Treatment of the necrotic head of the femur in adults. JBJS. 1949;31(1):55-66. 
[7] Rosenwasser MP, Garino JP, Kiernan HA, Michelsen CB. Long Term Followup of Through Debridement and Cancellous Bone Grafting of the Femoral Head for Avascular Necrosis. Clin Orthop Relat Res. 1994;306:17-27.

[8] Song WS, Yoo JJ, Kim Y-M, Kim HJ. Results of multiple drilling compared with those of conventional methods of core decompression. Clin Orthop Relat Res. 2007;454:139-146.

[9] Urbaniak JR, Coogan PG, Gunneson EB, Nunley JA. Treatment of osteonecrosis of the femoral head with free vascularized fibular grafting. A long-term follow-up study of one hundred and three hips. J Bone Joint Surg Am. 1995;77(5):681-694.

[10] Sugioka Y, Hotokebuchi T, Tsutsui H. Transtrochanteric anterior rotational osteotomy for idiopathic and steroid-induced necrosis of the femoral head. Indications and long-term results. Clin Orthop Relat Res. 1992;(277):111120.

[11] Mont MA, Zywiel MG, Marker DR, McGrath MS, Delanois RE. The natural history of untreated asymptomatic osteonecrosis of the femoral head: a systematic literature review. JBJS. 2010;92(12):2165-2170.

[12] Lieberman JR, Berry DJ, Montv $\mathrm{MA}$, et al. Osteonecrosis of the hip: management in the twenty-first century. JBJS. 2002;84(5):834-853.

[13] Petrigliano FA, Lieberman JR. Osteonecrosis of the hip: novel approaches to evaluation and treatment. Clin Orthop Relat Res. 2007;465:53-62.
[14] Markatos K, Savvidou OD, Foteinou A, et al. Hallmarks in the History and Development of Total Hip Arthroplasty. Surg Innov. Published online 2020:1-4. doi:10.1177/1553350620947209

[15] Trinkaus E. Pathology and the posture of the La Chapelle-aux-Saints Neandertal. Am J Phys Anthropol. 1985;67(1):19-41.

[16] Park H. An Account of a New Method of Treating Diseases of the Joints of the Knee and Elbow: In a Letter to Mr. Percival Pott. J. Johnson; 1733.

[17] Barton JR. On the treatment of anchylosis, by the formation of artificial joints. London Med Phys J. 1827;3(14):138.

[18] Gomez PF, Morcuende JA. Early attempts at hip arthroplasty--1700s to 1950s. Iowa Orthop J. 2005;25:25-29.

[19] Stiles PJ. Anthology of Orthopaedics, by Mercer Rang, Edinburgh and London, E. \& S. Livingstone, 1966, pp. xi, 243, illus., 42s. Med Hist. 1967;11(1):105-106.

[20] Markatos K, Sardiniari S, Brilakis E, Apostolopoulos I, Tsoucalas G, Chronopoulos E. Jules Pean (18301898)-A Pioneer Surgeon: His Achievements and His Total Shoulder Arthroplasty. Surg Innov. 2019;26(6):763765.

[21] Baer WS. a preliminary report of the use of animal membrane in producing mobility in ankylosed joints. jbjs. 1909;2(1):1-21 
[22] Baer WS. Arthroplasty with the aid of animal membrane. JBJS. 1918;2(2):94115.

[23] Smith-Petersen MN. Evolution of mould arthroplasty of the hip joint. J Bone Joint Surg Br. 1948;30(1):59-75.

[24] Girdlestone GR. The Classic: Acute Pyogenic Arthritis of the HipAn Operation Giving Free Access and Effective Drainage. Clin Orthop Relat Res. 1982;170:3-7.

[25] Kingston R, Walsh MG. The evolution of hip replacement surgery. Published online 2001.

[26] Moore AT, Bohlman HR. THE CLASSIC: Metal Hip Joint: A Case Report. Clin Orthop Relat Res. 2006;453:22-24.

[27] Thompson FR. Vitallium intramedullary hip prosthesis, preliminary report. $\mathrm{N}$ Y State J Med. 1952;52(24):3011.

[28] Wiles P. The surgery of the osteoarthritic hip. Br J Surg. 1958;45(193):488497.

[29] Amstutz HC. Innovations in design and technology: the story of hip arthroplasty. Clin Orthop Relat Res. 2000;378:23-30.

[30] Learmonth ID, Young C, Rorabeck C. The operation of the century: total hip replacement. Lancet. 2007;370(9597):1508-1519.

[31] Harris WH. The problem is osteolysis. Clin Orthop Relat Res. 1995;(311):46-53.
[32] Ranawat CS, Peters LE, Umlas ME. Fixation of the acetabular component: the case for cement. Clin Orthop Relat Res. 1997;344:207-215.

[33] Archibeck MJ, Jacobs JJ, Roebuck KA, Glant TT. The basic science of periprosthetic osteolysis. Instr Course Lect. 2001;50:185-195.

[34] Amstutz HC, Campbell P, Kossovsky N, Clarke IC. Mechanism and clinical significance of wear debrisinduced osteolysis. Clin Orthop Relat Res. 1992;(276):7-18.

[35] Maloney WJ, Jasty M, Harris WH, Galante JO, Callaghan JJ. Endosteal erosion in association with stable uncemented femoral components. J Bone Joint Surg Am. 1990;72(7):1025-1034.

[36] Vernon-Roberts B. The tissue response to total joint replacement prostheses. Sci basis Jt Replace. Published online 1977:86-129.

[37] Willert H, Semlitsch M. Reactions of the articular capsule to wear products of artificial joint prostheses. J Biomed Mater Res. 1977;11(2):157-164.

[38] Green TR, Fisher J, Stone M, Wroblewski BM, Ingham E. Polyethylene particles of a 'critical size'are necessary for the induction of cytokines by macrophages in vitro. Biomaterials. 1998;19(24):2297-2302

[39] Dumbleton JH, Manley MT, Edidin AA. A literature review of the association between wear rate and osteolysis in total hip arthroplasty. J Arthroplasty. 2002;17(5):649-661. 
[40] Kurtz SM. The UHMWPE Handbook: Ultra-High Molecular Weight Polyethylene in Total Joint Replacement. Elsevier; 2004.

[41] Dorr LD, Wan Z, Shahrdar C, Sirianni L, Boutary M, Yun A. Clinical performance of a Durasul highly crosslinked polyethylene acetabular liner for total hip arthroplasty at five years. JBJS. 2005;87(8):1816-1821.

[42] D'Antonio JA, Manley MT, Capello WN, et al. Five-year Experience with Crossfire (R) Highly Cross-linked Polyethylene. Clin Orthop Relat Res. 2005;441:143-150.

[43] Atienza Jr C, Maloney WJ. Highly cross-linked polyethylene bearing surfaces in total hip arthroplasty. J Surg Orthop Adv. 2008;17(1):27-33.

[44] Digas G, Kärrholm J, Thanner J, Herberts P. 5-year experience of highly cross-linked polyethylene in cemented and uncemented sockets: two randomized studies using radiostereometric analysis. Acta Orthop. 2007;78(6):746-754.

[45] Kurtz SM, Manley M, Wang A, Taylor S, Dumbleton J. Comparison of the Properties of Annealed Crosslinked (Crossfire $^{T M}$ ) and Conventional Polyethylene as Hip Bearing Materials. Bull Jt Dis. 2002;61(1-2):17-26.

[46] Dumbleton JH, D'Antonio JA, Manley MT, Capello WN, Wang A. The basis for a second-generation highly cross-linked UHMWPE. Clin Orthop Relat Res. 2006;453:265-271.

[47] Manley MT, Sutton K. Bearings of the future for total hip arthroplasty. J Arthroplasty. 2008;23(7):47-50.
[48] Simon JA, Dayan AJ, Ergas E, Stuchin SA, Di Cesare PE. Catastrophic failure of the acetabular component in a ceramic-polyethylene bearing total hip arthroplasty. J Arthroplasty. 1998;13(1):108-113.

[49] Semlitsch M, Willert HG. Clinical wear behaviour of ultra-high molecular weight polyethylene cups paired with metal and ceramic ball heads in comparison to metal-on-metal pairings of hip joint replacements. Proc Inst Mech Eng Part H J Eng Med. 1997;211(1):7388.

[50] Jazrawi LM, Kummer FJ, DiCesare PE. Alternative bearing surfaces for total joint arthroplasty. JAAOSJournal Am Acad Orthop Surg. 1998;6(4):198-203.

[51] Schmalzried TP, Peters PC, Maurer BT, Bragdon CR, Harris WH. Long-duration metal-on-metal total hip arthroplasties with low wear of the articulating surfaces. J Arthroplasty. 1996;11(3):322-331.

[52] Willert H-G, Buchhorn GH, Fayyazi A, et al. Metal-on-metal bearings and hypersensitivity in patients with artificial hip joints: a clinical and histomorphological study. JBJS. 2005;87(1):28-36.

[53] Boutin P. Arthroplastie totale de hanche par prothèse en alumine frittée. Rev chir orthop. 1972;58:229-246

[54] Kumar N, Arora NC, Datta B. Bearing surfaces in hip replacement Evolution and likely future. Med J Armed Forces India. 2014;70(4):371-376. doi:10.1016/j.mjafi.2014.04.015 
[55] Yang CC, Kim RH, Dennis DA. The squeaking hip: a cause for concerndisagrees. Orthopedics. 2007;30(9):739742.

[56] Ranawat AS, Ranawat CS. The squeaking hip: a cause for concernagrees. Orthopedics. 2007;30(9):738-743 